The Astrophysical Journal, 193:401-418, 1974 October 15

(C) 1974. The American Astronomical Society. All rights reserved. Printed in U.S.A.

\title{
AN INFRARED PHOTOMETRIC SURVEY OF PLANETARY NEBULAE
}

\author{
MARTIN COHEN \\ Astronomy Department, University of California, Berkeley \\ AND
}

Michael J. BARLOW

Astronomy Department, University of California, Berkeley, and Astronomy Centre, University of Sussex, England Received 1974 April 25; revised 1974 May 15

\section{ABSTRACT}

Infrared photometry between 2.2 and $22 \mu$ is presented of 113 planetary nebulae, covering a wide variety of types. Large excesses of flux are found at both 10 and $18 \mu$ in many nebulae, which are interpreted as being due to thermal emission by cool dust grains $\left(\sim 180^{\circ} \mathrm{K}\right)$. Several broad spectral features are revealed by the multifilter photometry of nine nebulae. An infrared discriminant is suggested between faint stellar objects which spectroscopically may be either unresolved planetary nebulae or reddened Be stars.

A very strong correlation between the existence of an infrared excess and the presence of an emission-line central star is found; in fact in two-thirds of the nebulae with emission-line nuclei observed an excess has been detected. Definite interdependences are found between nebular absolute 10- $\mu$ magnitudes and both electron densities and absolute fluxes at $10 \mathrm{GHz}$. A correlation between color temperature and electron density is also found. It is argued that these relations are consistent with $L \alpha$ heating of the grains. Infrared and ultraviolet luminosities of the nebulae are compared, and the origin of the grains is discussed. Evidence is given for the formation of dust in the mass-loss outflows from the emission-line nuclei of several planetary nebulae.

Subject headings: infrared — planetary nebulae

\section{INTRODUCTION}

There have been several photometric studies of planetary nebulae in the near-infrared $(1.0-3.5 \mu)$, which have indicated mostly that this radiation is due to free-free emission, although hot dust has been invoked to explain excesses found above free-free radiation in a few nebulae (Khromov and Moroz 1972; Willner, Becklin, and Visvanathan 1972, hereinafter referred to as WBV; Persson and Frogel 1973, hereinafter referred to as PF). Additionally, Gillett, Merrill, and Stein (1972, hereinafter referred to as GMS) made observations near $11 \mu$, using both a moderately broad filter to measure any continuum emission and a narrow filter centered at $10.52 \mu$ to isolate the S IV line. Most of the nebulae which they detected showed a true continuum which was substantially stronger than this line emission.

As part of a continuing investigation of the infrared properties of hot stars, this paper reports new observations of planetary nebulae, primarily at 10 and $18 \mu$, and offers evidence which supports an interpretation of this long-wavelength radiation as due to thermal reradiation by dust grains.

\section{SELECTION OF OBJECTS}

Smith and Aller (1969) have constructed a classification scheme for the emission-line spectra of the nuclei of planetary nebulae, based upon parallels with the spectra of Population I stars. Most of the nebulae whose nuclei they classified have been included in our survey. Whether some nuclei have emission-line or continuous spectra is uncertain, and several nebulae containing such stars have been observed. It further seemed important that observations of the brighter nebulae at $11 \mu$ detected by GMS be made at both 8.6 and $18 \mu$, to define more clearly the nature of their infrared continua. Again priority was given to nebulae whose stars possess emission lines, although several nebulae having nuclei with continuous spectra have been reobserved also.

As Allen (1973) has recently pointed out on the basis of near-infrared photometry, one of the vaguest boundaries in spectroscopic classification is that between the Be stars and the compact planetaries. A number of compact nebulae which may be more accurately termed $\mathrm{Be}$ stars have been included in this program, and it will be shown that the long-wavelength infrared energy distributions provide a powerful discriminant between these two classes of object. Sometimes confused with the Be stars are a number of very low-excitation planetary nebulae discussed by Sanduleak and Stephenson (1972a); several of these have been observed.

One of the types of spectrum described by Smith and Aller (1969) is the "O vi sequence," in which the very high-excitation O vi doublet $\lambda \lambda 3811,3834$ appears. Sanduleak (1972) has called attention to five stars, not known to be associated with planetaries, which show this doublet in emission. Because of their possible generic relationship to planetary nebulae (Sanduleak 1972), we observed the two stars accessible from the northern hemisphere.

A significant number of nebulae of miscellaneous type were included, whose principal merit was their accessibility during the spring months. Several compact nebulae for which radio fluxes greater than $0.1 \mathrm{Jy}^{1}$

$$
{ }^{1} 1 \text { jansky }=10^{-26} \mathrm{~W} \mathrm{~m}^{-2} \mathrm{~Hz}^{-1} \text {. }
$$


TABLE 1

Infrared Magnitudes of Five Standard Stars in the Multifilter Photometric System

\begin{tabular}{|c|c|c|c|c|c|c|c|c|c|c|}
\hline Star & [2.2] & [3.6] & [4.8] & [8.6] & [10] & [10.8] & [11.3] & {$[12.8]$} & [18] & [22] \\
\hline 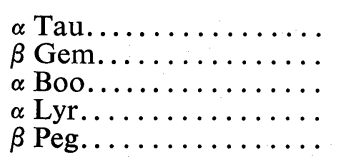 & $\begin{array}{l}-2.84 \\
-1.12 \\
-3.03 \\
0.0 \\
-2.24\end{array}$ & $\begin{array}{l}-3.02 \\
-1.19 \\
-3.21 \\
0.0 \\
-2.45\end{array}$ & $\begin{array}{l}-2.82 \\
-1.14 \\
-3.07 \\
-0.02 \\
-2.27\end{array}$ & $\begin{array}{l}-2.97 \\
-1.29 \\
-3.20 \\
-0.03 \\
-2.45\end{array}$ & $\begin{array}{l}-3.00 \\
-1.30 \\
-3.30 \\
-0.03 \\
-2.50\end{array}$ & $\begin{array}{l}-2.98 \\
-1.30 \\
-3.25 \\
-0.03 \\
-2.50\end{array}$ & $\begin{array}{l}-2.99 \\
-1.33 \\
-3.27 \\
-0.03 \\
-2.50\end{array}$ & $\begin{array}{l}-3.00 \\
-1.30 \\
-3.30 \\
-0.03 \\
-2.50\end{array}$ & $\begin{array}{r}-3.1 \\
-1.3 \\
-3.4 \\
0.0 \\
-2.5\end{array}$ & $\begin{array}{r}-3.1 \\
-1.3 \\
-3.4 \\
0.0 \\
-2.5\end{array}$ \\
\hline
\end{tabular}

were indicated by the 15.4-GHz survey of Rubin (1970) were also included. The selection of all these objects tends to reduce the bias in our overall sample, and provides some information on otherwise underobserved nebulae.

The object VV 57, mentioned by Smith and Aller (1969), was not observed in this survey since it is the ring nebula NGC 2359, excited by the Population I Wolf-Rayet star HD 56925 of type WN 5.

\section{THE OBSERVATIONS}

\section{a) Magnitudes}

Virtually all nebulae were observed using the UM-UCSD 1.5-m telescope on Mount Lemmon, Arizona. The program was initiated in 1972 December and continued until 1974 April. Mostly wide bandpass filters were used $(6 \mu$ centered at $10 \mu ; 4 \mu$ centered at $18 \mu$ ) at long wavelengths, but the photometer incorporated both broad and narrow filters, thereby permitting a very flexible approach to the acquisition of data. Details of this multifilter photometric system are given by Cohen (1973a). Table 1 presents the multifilter magnitudes of five standard stars. Throughout the program, 11" beams with a 15" throw were used at Mount Lemmon. Due to the northerly restriction on the UM-UCSD telescope, the observations of 5 objects were made with the $3.05-\mathrm{m}$ reflector of the Lick Observatory; $4^{\prime \prime}$ beams with an $8^{\prime \prime}$ throw were used.

Tables 2 and 3 present our observations; table 2 contains principally those nebulae for which observations were made at only a few wavelengths, while table 3 comprises multifilter observations. Each table gives the name of the nebula, its number in the Catalog (CGPN) of Perek and Kohoutek (1967) and the spectral type of the nucleus (following Aller 1968, and Smith and Aller 1969). Existing short-wavelength photometry from a variety of authors is included for completeness (WBV; PF; Khromov and Moroz 1972; Allen 1973, 1974a; Allen and Glass 1974), as are 11- $\mu$ data by GMS.

In order to improve the accuracy of measurements and test the reproducibility of the faintest magnitudes, a number of nebulae have been observed on more than one occasion. It is found that $10-$ and $18-\mu$ magnitudes brighter than $\sim+4.5$ and $\sim+0.9$, respectively, have been reproduced within $1 \sigma$ of the mean. Objects fainter than these limits are reproducible within 1.0 $1.5 \sigma$ and invariably are detected as the result of long periods of integration.

\section{b) Notes on Individual Objects}

During this survey, the objects Sh2-266, containing the emission-line star MWC 137, M1-67, and NGC 2346 were observed. Their overall properties seem so different from those of planetary nebulae that they will be discussed elsewhere (Cohen and Barlow 1974).

M2-9 $\left(10+18^{\circ} 2\right)$. - A full discussion of this peculiar object is given by Allen and Swings (1972a). Our energy distribution (fig. 3) indicates a curve much broader than a blackbody, peaking near $8 \mu$; the corresponding Planckian peak temperature is $\sim 450^{\circ} \mathrm{K}$. Allen and Swings $(1972 b)$ find an $(H-K)$ color temperature of $\sim 830^{\circ} \mathrm{K}$. Clearly no unique temperature can be ascribed to this object. The radio observations by Purton and Feldman (1974) indicate that the radio emission comes from the extended wings of this nebula, rather than from the dense infrared core. The very large ratio of infrared to $L \alpha$ luminosity which we have derived ( $\sim 80$; see table 4$)$ supports the view that this object is not a genuine planetary nebula.

IC $4997\left(58-10^{\circ} 1\right)$. - Gillett, Knacke, and Stein (1971) observed this compact nebula, and found $[11.0]=+2.7 \pm 0.1$ using a $5^{\prime \prime}$ beam. The size of the nebula is given as $\sim 2^{\prime \prime}$ by the CGPN, yet our observations with an $11^{\prime \prime}$ beam yield $[11.3]=+2.3 \pm 0.2$. There is photometric evidence that this object is variable (Kostjakova, Arhipova, and Savel'eva 1973). It is possible that the variability extends to the $10-\mu$ region, and it would be interesting to monitor this nebula at long wavelengths.

He 1-5 = FG Sge $\left(60-7^{\circ} 1\right)$. - This is a well-studied peculiar object, perhaps representing an early phase in the life of a planetary nebula (Herbig and Boyarchuk 1968; Arhipova 1973; Herbig and Flannery 1973). Near-infrared magnitudes were obtained by Gillett et al. (1971) $([3.5]=+7.1)$ and by Allen (1973) $([1.6]=$ $+7.3,[2.2]=+7.1)$. Allen noted that the object was slowly brightening at $2 \mu$. Our magnitudes at $2.2 \mu$ $(=6.8)$ and $3.6 \mu(=6.5)$ indicate that this brightening is continuing, by comparison with the earlier measurements. Since the visual magnitude of the star has been fairly constant during this period, the brightening at $2 \mu$ is presumably due to the evolution of the star toward later spectral type (Arhipova 1973). There is no evidence of an appreciable near-infrared excess.

M1-76 $\left(74+1^{\circ} 1\right)$. - Both Bidelman and Krumenaker (1972) and Allen (1974a) find that spectra of this object are not typical of conventional planetary nebulae; in particular, each finds an appreciable continuum. Bidelman and Krumenaker note the presence of a 
strong emission line of $\mathrm{O}_{\mathrm{I}} \lambda 8446$ which is found in a number of Be stars, and suggest that M1-76 may be a highly reddened star of the P Cygni type. This interpretation is consistent with our long-wavelength data which indicate a free-free slope (see fig. 2 and $\S$ IV), although the near-infrared indices definitely indicate the presence of dust emission.

M2-56 $\left(118+8^{\circ} 1\right)$. - Only two objects from the CGPN are included in the AFCRL infrared sky survey, NGC 7027 and M2-56, the former identified and the latter hitherto unrecognized. The coordinates of one AFCRL object apparently matched those of M2-56, albeit with large uncertainties, and the " $1 \sigma$ error box" of the rocket source was scanned at $10 \mu$ in 1973 December with the Lick 3.05-m reflector. The tentative identification of M2-56 was confirmed, with good agreement between the fluxes at $10 \mu$ in a $4^{\prime \prime}$ diaphragm and the rocket aperture $\left(10^{\prime} \times 3^{\prime}\right)$, implying the pointlike nature of the source. M2-56 is an exceedingly faint object, being invisible without long integration periods through the Cassegrain TV guiding system at Lick. Its red magnitude is consequently $\geqslant 18$, so that the energy distribution rises extremely rapidly between the red and the near-infrared. Allen (1974a), too, has noted the sharp rise in its spectrum between 1.6 and $3.5 \mu$.

On the Palomar Sky Survey prints, the object is certainly nonstellar in the red, having a nebulous, elliptical appearance of dimensions approximately $8^{\prime \prime} \times 5^{\prime \prime}$ with the major axis extended roughly EW. No nucleus is apparent. It is essentially invisible on the blue print. Minkowski's (1974) original spectrum revealed only the presence of $\mathrm{H} \alpha$ emission with no continuum, although Allen (1974a) has reported the presence of other emission lines.

Kohoutek (1972) felt that the object was brighter at the time of its discovery (before 1947) than at present, and thought that it might be a variable star or perhaps a nova. Its faintness in the Lick TV system suggests that it is fainter now than it appears on the Palomar red print, confirming Kohoutek's impression of dimming. However, its symmetrical nebulous appearance is not typical of any known group of variable stars.

IC $3568\left(123+34^{\circ} 1\right)$. - The O-type nucleus of this nebula has $V=11.39,(B-V)=0.67$, and $(U-B)=$ - 0.56 (Kostjakova et al. 1968). Since it lies at $b^{\text {II }}=+34^{\circ}$, it would appear that this large amount of reddening is internal to the nebula. Based upon existing radio data at $5 \mathrm{GHz}$ (Johnson 1973), the predicted value of the free-free emission at $10 \mu$ is $0.05 \mathrm{Jy}$. Our observations with a $4^{\prime \prime}$ beam yield a value of $0.22 \mathrm{Jy}$ (two independent detections at this value). Structurally this nebula consists of a bright, dense inner core $\left(\sim 6^{\prime \prime}\right)$ surrounded by a much fainter outer region $\left(\sim 18^{\prime \prime}\right)$. It is possible that most of the free-free emission originates from this inner core so the $10-\mu$ detection of this object implies a substantial excess of radiation over free-free, which might be attributed to thermal emission by dust.

The spectrum of the nucleus has been described as an absorption line O5 star (Aller 1968). However, an emission line of $\mathrm{C}$ IV seen in the spectrum of the nebula may be due to the central star, according to Aller and Walker (1970). If the star has an absorptionline spectrum we would not expect to find dust in the nebular core, whereas if it is an emission-line star we would expect dust to be present (see $\S$ VI). Indeed, the $U B V$ data imply much reddening, yet Lee et al. (1969) noted no disagreement between the observed and predicted Balmer decrements for this nebula, suggesting no appreciable internal space absorption. It is worthy of note that Johnson (1973) has detected an abnormally large flux at $85 \mathrm{GHz}$ from IC 3568 .

$N G C 1514\left(165-15^{\circ} 1\right)$. - The nucleus of this nebula has a composite spectrum, classified as A0 III + a blue subdwarf by Kohoutek (1968). Greenstein (1972) showed the blue subdwarf to be an $\mathrm{O}$ star, whose parameters were typical of a normal planetary nucleus. Shao and Liller (1974) give $V=9.42,(B-V)=$ 0.55 ; and Kostjakova et al. (1968) give $V=9.64$, $(B-V)=0.53$. Since Greenstein (1972) gave $M_{V}$ for the A star as +0.8 and for the $\mathrm{O}$ star as +2.8 , the $B$ and $V$ data clearly refer to the A star. The data of Kostjakova et al. yield $V_{0}=8.05$, and hence [2.2] $=$ 8.22 and $[3.6]=8.13$, using the reddening corrections computed by Lee (1968). There is thus no infrared excess at 2 or $3 \mu$ by comparison with our observations (table 2).

$N G C 2392\left(197+17^{\circ} 1\right)$. - This planetary has a nucleus which is the sole representative of the "Of" subclass of Smith and Aller (1969), corresponding to the classical Population I Of stars. Our limits at $3.6 \mu$, combined with the preexisting 1.6- and 2.2- $\mu$ data, exclude the presence of hot $\left(\sim 1000^{\circ} \mathrm{K}\right)$ dust in the nebula, while the limits at 10 and $18 \mu$ make it unlikely that much cool dust is present.

$V V 1-7\left(235+1^{\circ} 1\right)$. - The nucleus of this nebula, BD $-18^{\circ} 1967$, is a B9 star of $V=8.31,(B-V)=$ 0.10 (Kostjakova et al. 1968). Thus our values of [2.2] and [3.6] in table 2 are consistent with there being no near-infrared excess.

Sanduleak 4.-This star is one of the five stars showing O vi emission discussed by Sanduleak (1972), and it lies within a $5^{\prime}$ ring nebula. The broad emission of He II $\lambda 4686$ and the O vI doublet of Sanduleak 4 indicate expansion in the range $8000-10,000 \mathrm{~km} \mathrm{~s}^{-1}$ (Blanco, Kunkel, and Hiltner 1968). With $V=13.4$, $(B-V)=+1.0$ (Blanco et al. 1968) this star appears to be intrinsically much brighter than the $O$ vi nuclei of planetary nebulae. Consequently, our failure to detect both Sanduleak 4 and 5 at $10 \mu$ may indicate a difference between Population I and Population II $O$ vi stars (two of Sanduleak's five stars lie in the Magellanic Clouds, indicating a very high luminosity). It should be noted, however, that Johnson (1973) has once again found a remarkably high $85-\mathrm{GHz}$ flux from the nebula, so that far-infrared observations of this object could be most valuable.

HD 316285.-Following Allen (1973) we observed this object, believing it to be B1 3-11(1-0 1$)$. However, the CGPN indicates a starlike object nearby to be the planetary. No signal was found from this planetary, but a large $10-\mu$ signal was detected from the star HD 316285. According to Allen (1974b), HD 316285 has 


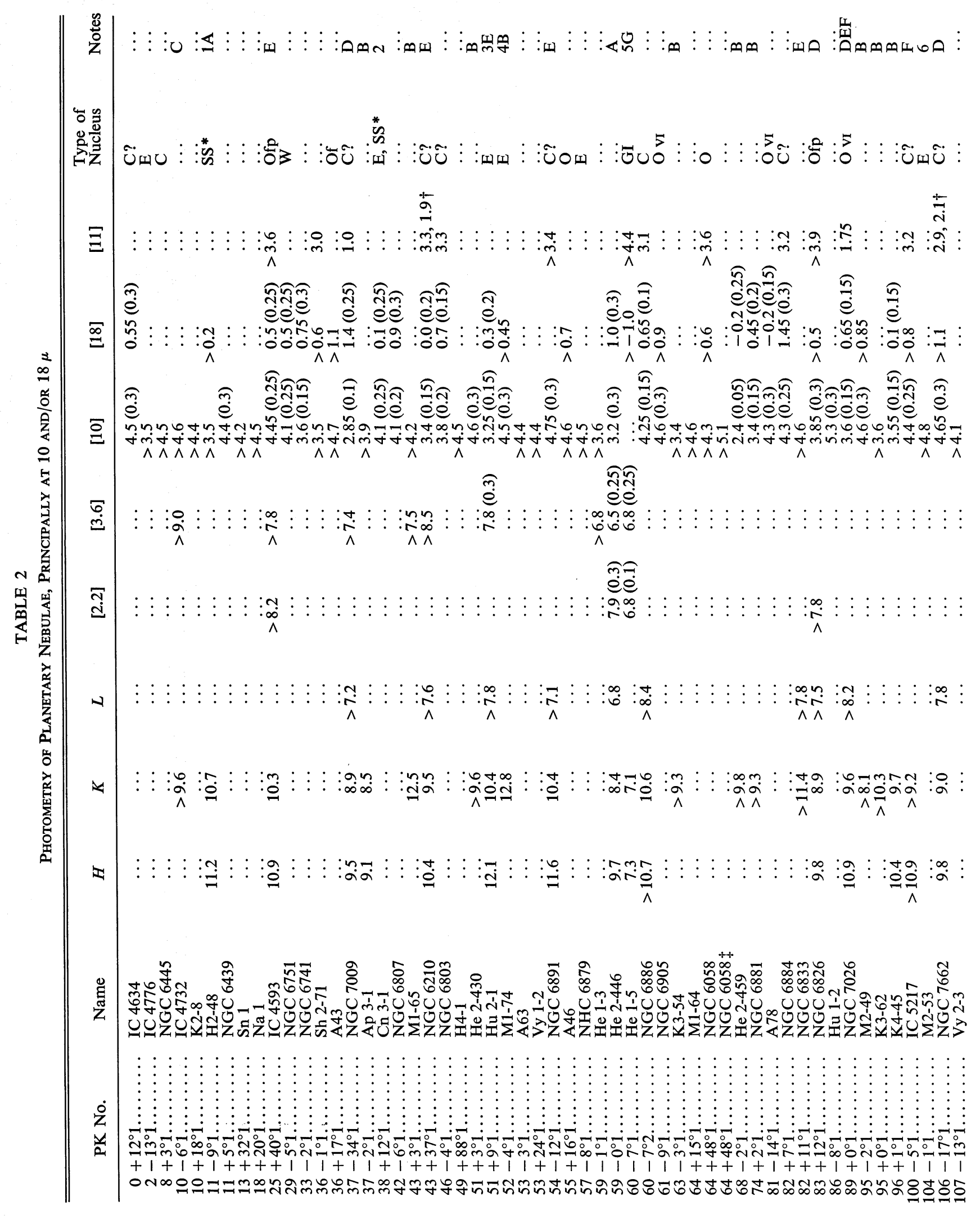




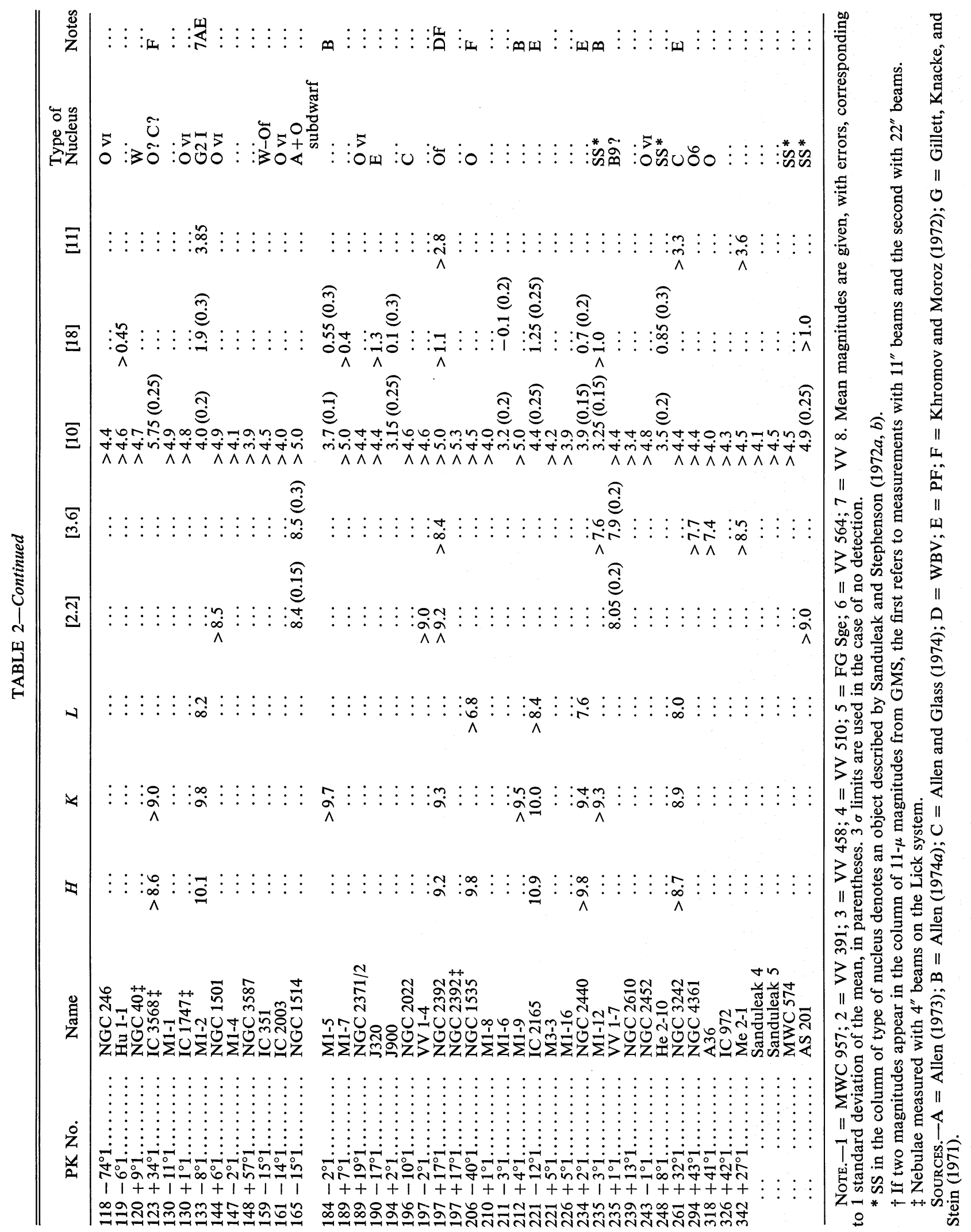

405 


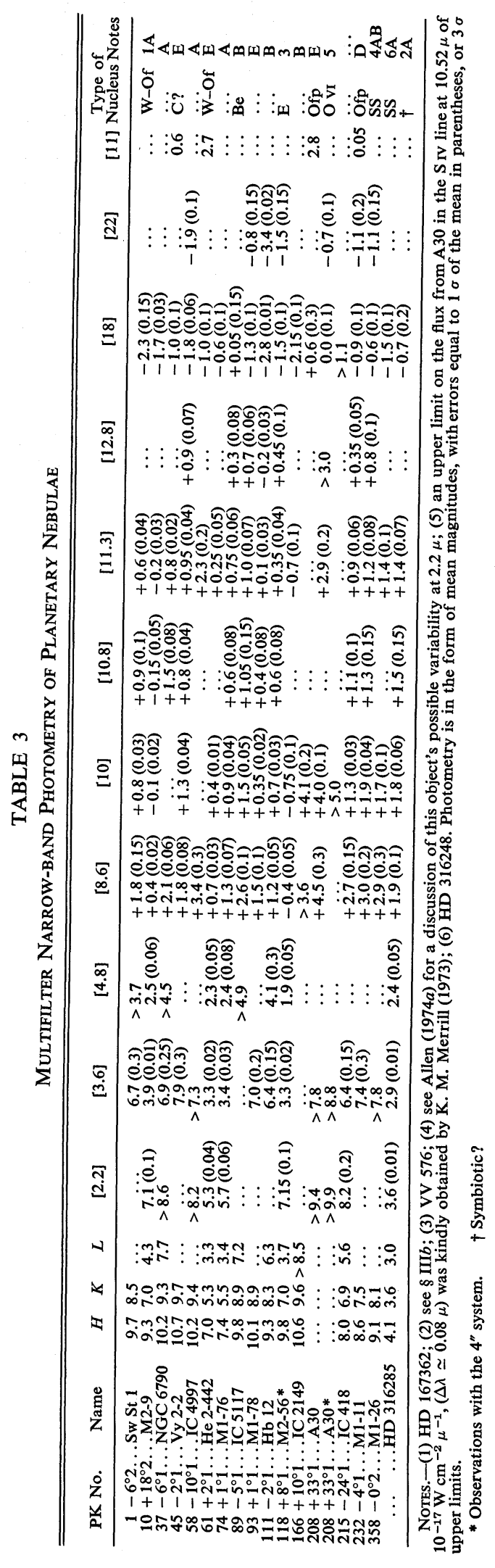

(C) American Astronomical Society - Provided by the NASA Astrophysics Data System 
strong $\mathrm{H} \alpha$ emission whereas none is visible from $\mathrm{Bl}$ 3-11, making it possible that the HD star is in reality the emission-line object found by Blanco. The spectral type given in the Henry Draper Catalog is "peculiar," and the star may be a symbiotic star. Consequently it may not be typical of planetaries in its energy distribution, although the initial slope of the spectrum at short wavelengths (fig. 2) does give way to an increased flux level between 10 and $18 \mu$, though less dramatic than that found for many planetaries. This should be compared with the same type of rise at the longest wavelengths in the symbiotic star Z And (Woolf 1973).

$A S$ 201.-This star is one of the objects described by Sanduleak and Stephenson (1972b), as having strong O III $\lambda 4363$ emission. It resembles the object M1-2 (see fig. 2) in this respect and in having an associated G-type spectrum. Both may be relevant to protoplanetary objects, so that our detection of AS 201 at $10 \mu$ is significant.

\section{c) The Spatial Distribution of Infrared Radiation in Planetaries}

Many of the nebulae we have observed are smaller than our $11^{\prime \prime}$ beam size; in these cases no statement can be made as to the distribution of the radiation. Several nebulae are larger than our beams and comparison of our data with the previous observations by GMS with a $22^{\prime \prime}$ beam indicates definite areal effects. GMS observed some nebulae themselves with $11^{\prime \prime}$ and $22^{\prime \prime}$ diaphragms and found this effect at $11 \mu$ (e.g., for NGC 6537, NGC 6572). IC 418 is a good example of the fact that infrared emission at a range of wavelengths may be found outside the optical boundaries of some nebulae. Our 2.2- and 3.6- $\mu$ magnitudes (each measured on two independent occasions) are significantly fainter than data taken with beams much larger than the nominal diameter $\left(\sim 12^{\prime \prime}\right)$ of the ionized region (see table 3 ). Further, comparison of our 10.8and $11.3-\mu$ data for this nebula with the $11-\mu$ observations of Gillett and Stein (1969) with a 16" diaphragm may also suggest the presence of infrared radiation beyond the optical nebulosity. Inspection of tables 2 and 3 reveals that for many of the larger planetaries not all of their infrared flux is likely to have been detected with our beams. However, it seemed important for very large objects like Abell 30 to establish whether the apparent concentration of radiation on the nucleus was really just a small feature within an extended source. Therefore, efforts were made to "map" this nebula at 10 and $18 \mu$.

The first indication that the signal from the nucleus of A30 is not from a point source comes from direct comparison of the $11^{\prime \prime}$ photometry with that from the Lick $4^{\prime \prime}$ system. As table 3 reveals, not even $3 \sigma$ detections of A30 were obtained at 10 or $18 \mu$ with the 3.05-m telescope, although signals are easily detected with the $1.5-\mathrm{m}$ Mount Lemmon telescope. These $3 \sigma$ limits are each brighter than the empirical threshold of detectability at Lick. Marginal $(\sim 2 \sigma)$ positive signals were found with the $4^{\prime \prime}$ beams at flux levels some 5 times less than those derived from the $11^{\prime \prime}$ photometry. One concludes, therefore, that an extended source $\left(>4^{\prime \prime}\right)$ is present at long wavelengths, though it is perhaps not of uniform brightness over a diameter $\geqslant 11^{\prime \prime}$, since this would imply that the flux in the small beam should be no more than $(4 / 11)^{2} \simeq 1 / 8$ of that in the larger beam.

The emission at 10 and $18 \mu$ along two diameters through the nucleus of A30 (one N-S, the other E-W) was mapped from Mount Lemmon on several occasions in the period 1974 January to April. The "map" data consist of integrations at a series of points along each diameter, in units of $\pm 15^{\prime \prime}$ from the nucleus. These maps were extended beyond the optical rim of the nebula in each direction. The total accumulated integration time per point is approximately 25 minutes for the $10-\mu$ map and 15 minutes for the $18-\mu$ map, the latter being intrinsically easier, due to the steep spectrum of A30 and the smaller sky emission at $18 \mu$.

Definite gradients are present in the emission profiles, at each wavelength, close to the nucleus, but the emission levels are not significantly different from zero at points beyond $30^{\prime \prime}$ from the central star. Each profile (N-S and E-W) has a FWHM of $\sim 25^{\prime \prime}$ at 10 and $18 \mu$. No significant "hot spots" were encountered in the map except for the nucleus, nor any indication of increased emission toward the optical edge of the nebula.

The infrared radiation of A30 would appear to derive from the immediate environment of the central star, although the emission region is slightly extended $\left(\sim 30^{\prime \prime}\right.$ in diameter).

\section{ENERGY DISTRIBUTIONS}

The energy distributions of 20 objects are presented in figures 1-4, with ordinates $\lambda F_{\lambda}\left(\mathrm{W} \mathrm{cm}^{-2}\right)$ and

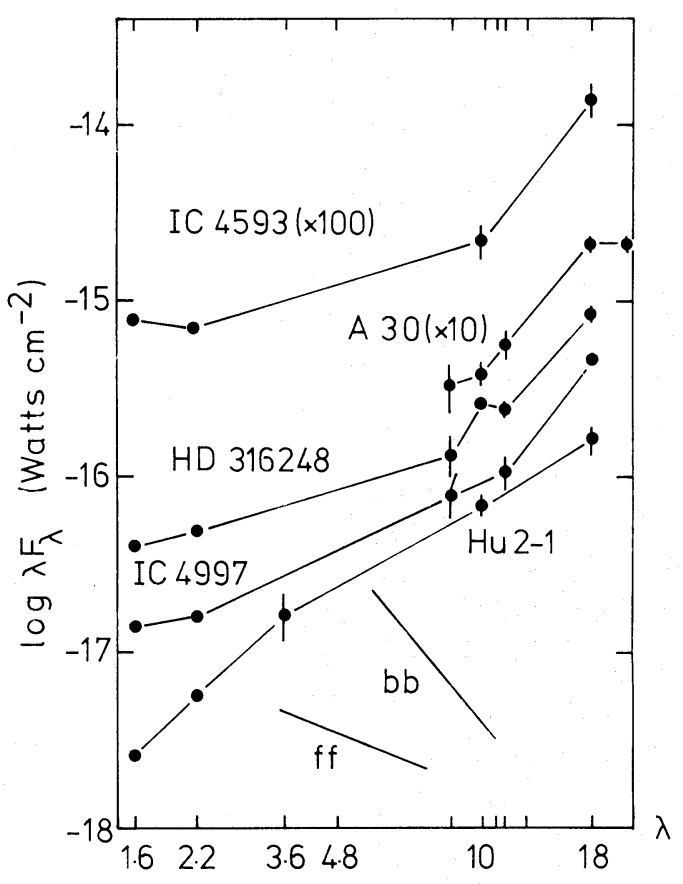

FIG. 1.-Energy distributions of planetary nebulae 


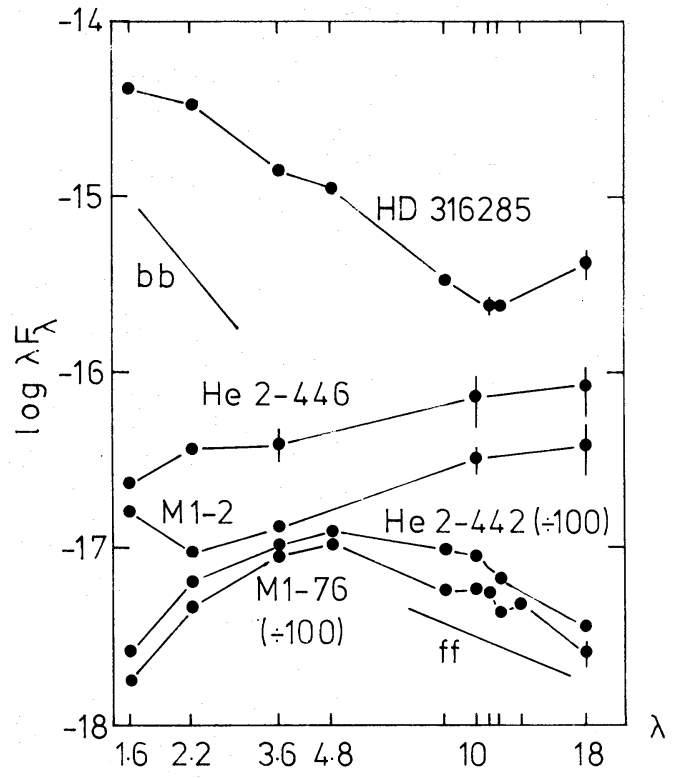

FIG. 2.-Energy distributions of emission-line objects, including a possible symbiotic star (HD 316285), the peculiar object M1-76, and the protoplanetary nebula M1-2.

abscissae $\lambda(\mu)$, each plotted logarithmically. These have been grouped according to the overall shapes of the distributions. Figure 1 comprises principally those objects with flat or slowly rising spectra in the nearinfrared, which rise quite steeply at long wavelengths. Figure 2 includes three types of distribution: HD 316285 (see $\S \mathrm{III} b$ ) may be a symbiotic star and, apart

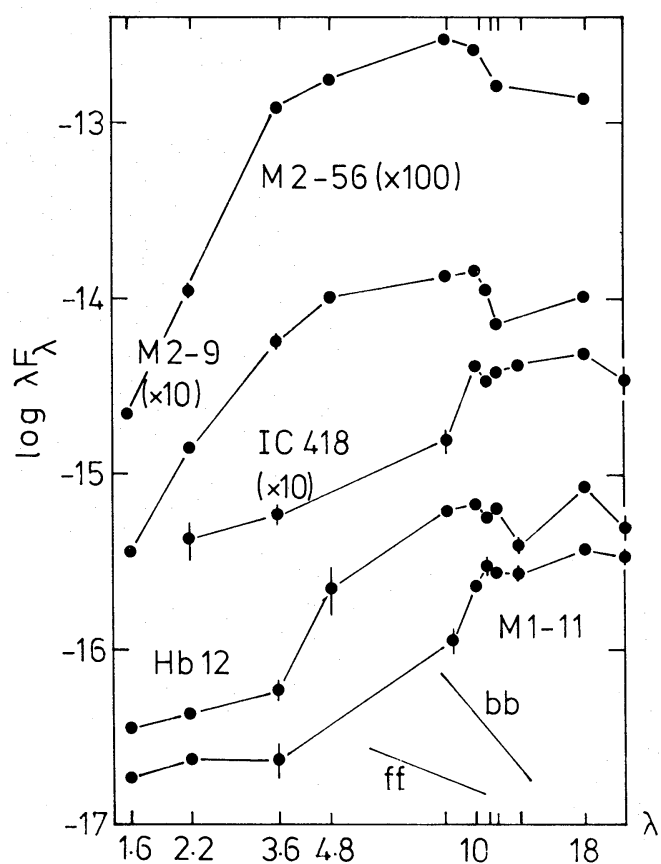

FIG. 3.-Spectra of planetary nebulae which are relatively flat at long wavelengths.

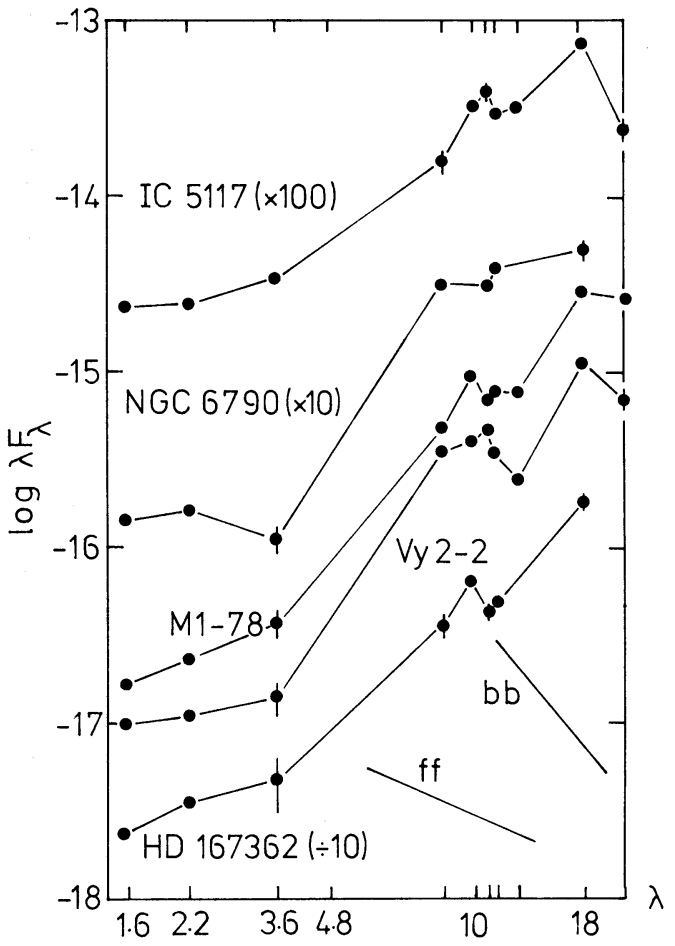

FIG. 4.-Steeply rising spectra of planetary nebulae

from the unexpected rise at $18 \mu$, is probably not representative of planetary nebulae; two rather flat spectra (M1-2 and He 2-446); two curves which have a broad peak $\sim 5 \mu$ and which asymptotically decline to an optically thin free-free slope between 10 and $18 \mu$. Figure 3 contains objects whose spectra rise quite steeply from short wavelengths yet which have flattish distributions in the 8-22 $\mu$ region. Finally, figure 4 includes distributions which rise very steeply for $\lambda \geqslant 3.6 \mu$, and which in general continue to rise from 10 to $18 \mu$. Each figure includes for comparison the slopes of the Rayleigh-Jeans region of a blackbody (" $\mathrm{bb}$ "; $\lambda F_{\lambda} \propto 1 / \lambda^{3}$ ) and of optically thin free-free emission ("ff"; $\lambda F_{\lambda} \propto 1 / \lambda$ ).

It should be noted that in some nebulae with very steeply rising curves, the broad $10-\mu$ point fits rather poorly with the narrow-band data. This is probably due to the effective wavelength of this wide bandpass filter being somewhat longward of $10 \mu$, when convolved with such a spectrum. Some features appear in the $10-\mu$ region, but none which clearly resemble the "silicate" emission signature so common among latetype stars (IC 5117 is perhaps the closest in general structure to these).

It is evident that a variety of energy distributions are found and that although observations at 2 and $3 \mu$ will isolate such objects as $\mathrm{Hu} 2-1$ and M1-76, 10- $\mu$ observations are crucial to the picture of planetary nebulae. In particular, we have detected several objects at 10 and/or $18 \mu$ which did not yield detections to Allen's (1974a) recent 2.2- $\mu$ survey (e.g., M1-5, K3-62, He 2-459, He 2-430, NGC 6881). 
The only objects which mimic the free-free slope in their $\lambda F_{\lambda}$ plots (again excluding HD 316285) do so at long wavelengths; these are He 2-442 and M1-76 (see $\S$ III $b)$. We feel that the steep rise, or decline, of $\lambda F_{\lambda}$ from 10 to $18 \mu$ may provide a powerful discriminant between virtually stellar or unresolved nebulae (such as HD 167362, Vy 2-2) and Be stars (e.g., M1-76), particularly in those cases when forbidden lines are not clearly present on objective prism plates or when the existence of a continuum is suspected.

Several questions arise immediately concerning the processes producing the infrared radiation of planetary nebulae. Do we see continuum or line radiation? If continuum, is it merely an extrapolation of the freefree radiation detected at radio frequencies? Could thermal emission by cool dust grains account for the brightness of these objects, especially at $18 \mu$ ?

Andrillat (1968) has listed those (forbidden) lines which are relevant to the infrared region in planetary nebulae. For several nebulae observations exist with the narrow-band filters within the $10-\mu$ region and emission is found in all these filters. This fact, coupled with the considerable line strengths required to explain detections near $10 \mu$ when degraded into large bandpass filters, makes it highly improbable that the frequency with which both 10- and larger 18- $\mu$ fluxes occur in diverse nebulae is explicable in terms of line emission. GMS found that the $10.52-\mu \mathrm{S}$ IV line was only a significant contributor in a few instances to the total $11-\mu$ emission of the nebulae they studied. While it is entirely possible that the S IV $10.52-\mu$ line and/or the Ne II 12.8- $\mu$ line contribute somewhat to the $10-\mu$ fluxes, it appears that continuum emission is certainly present in many nebulae. Gillett, Forrest, and Merrill (1973) have discovered a broad emission feature centered at $11.3 \mu$ in the spectra of NGC 7027 and BD $+30^{\circ} 3639$. If such a feature were present in nebulae for which we have obtained $11.3-\mu$ broad-band data, at the same contrast level with respect to the continuum as in NGC 7027, it would be difficult to recognize. The spectra of Gillett et al. (1973) also rule out the possibility of a superposition of lines producing the $10-\mu$ radiation in two of the brightest of all nebulae.

We have calculated the $10-\mu$ flux due to an entire nebula to be expected from an extrapolation of that seen in the radio region, assuming that this latter derives from free-free emission. Information on the radio spectra of nebulae, for example from Higgs (1971), enables us to select a radio frequency at which the free-free emission is optically thin, and the flux at $10 \mu$ is computed on the assumption that an optically thin extrapolation from this frequency is valid. Appropriate Gaunt factors were used and we assumed that the maximum in the free-free spectrum had not been reached by $10 \mu$ (i.e., that $T_{e}>1440^{\circ} \mathrm{K}$, which is borne out by radio data). For several nebulae which are smaller than our beam, the ratio of $(10 \mu$ observed flux $/ 10 \mu$ flux expected from free-free emission) is in the range 8-100. This is in broad agreement with the earlier findings of GMS, who found ratios between 25 and 160. Thus, although an extrapolation of the freefree emission seen in the radio may explain the brightness of some planetary nebulae at 2.2 and $3.6 \mu$ (WBV; PF; Allen 1973), this is not in general an important contributor at 10 or $18 \mu$.

The steepness of the energy distributions at long wavelengths together with the presence of apparent features near $10 \mu$ is strongly suggestive of thermal emission by cool dust grains, and the interpretation of the radiation offered in the remainder of this paper will be in terms of this process.

\section{NEBULAR PARAMETERS AND HEATING MECHANISMS}

\section{a) Parameters}

Table 4 lists various parameters for all those nebulae which have been detected at $10 \mu$ and for which distance estimates exist in the catalog of Higgs (1971). These distances, based upon a model for the radiofrequency emission from planetary nebulae (Higgs 1973), have been adopted since they are independent of reddening estimates and because the model yields a consistent sequence of $10-\mathrm{GHz}$ flux estimates, upon which our calculation of $L \alpha$ luminosities was based.

Case II of Higgs (1971) has been taken, in which a standard nebular temperature is assumed for the derivation of a predicted $10-\mathrm{GHz}$ flux, electron density, and distance. Using these parameters we have calculated the following quantities: (1) $F_{\text {abs }}(10 \mathrm{GHz})$, the flux which the nebula would have if it were at a distance of $10 \mathrm{pc}$; (2) $M_{10}$, the absolute $10-\mu$ magnitude of the nebula; (3) $L_{\mathrm{L} \alpha}$, the nebular $\mathrm{L} \alpha$ luminosity, derived using the formula of Rubin (1968) for the total number of ionizing photons emitted by the exciting star per second, multiplied by the energy of a $\mathrm{L} \alpha$ photon, where every ionizing photon is assumed to lead to one $\mathrm{L} \alpha$ photon; (4) $L_{\mathrm{IR}}$, the infrared luminosity. To obtain the infrared luminosity, our data were combined with other short-wavelength photometry (see tables 2 and 3 ) yielding the spectral intensity $\left(F_{\lambda}\right)$ curves in the range $\lambda \lambda 1.6-22 \mu$. Integration beneath these curves produced the quantities $L_{\mathrm{IR} \text {, observed }}$ and by allowing for the significant amount of radiation longward of our last observed wavelength, corrections $\Delta L_{\infty}$ (Cohen 1973b) were made. $L_{\mathrm{IR}}$ is the sum of $L_{\mathrm{IR} \text {, observed }}$ and $\Delta L_{\infty}$, and should provide a good lower limit to the bolometric luminosity of the nuclei.

Whenever possible, a 10- $\mu$ magnitude was measured and this has been used in preference to preexisting measurements in constructing energy distributions and in evaluating infrared luminosities. For five nebulae not reobserved by us, for which narrowband data near 8 and $11 \mu$ were available, $10-\mu$ magnitudes were computed by requiring that the value of $F_{\lambda}$ at $10 \mu$ should be the average of $F_{\lambda}$ at 8 and $11 \mu$. Where there existed large-beam observations near $10 \mu$ of nebulae whose diameters exceed our beam size, the values of $M_{10}$ tabulated are those derived from the largest aperture measurements and the scaling factors to the $10-\mu$ flux for aperture size were applied to our $18-\mu$ data (if available) to produce an $L_{\mathrm{IR}}$ appropriate to the entire nebulae. This implicitly assumes that the color temperatures are uniform across these large nebulae. 


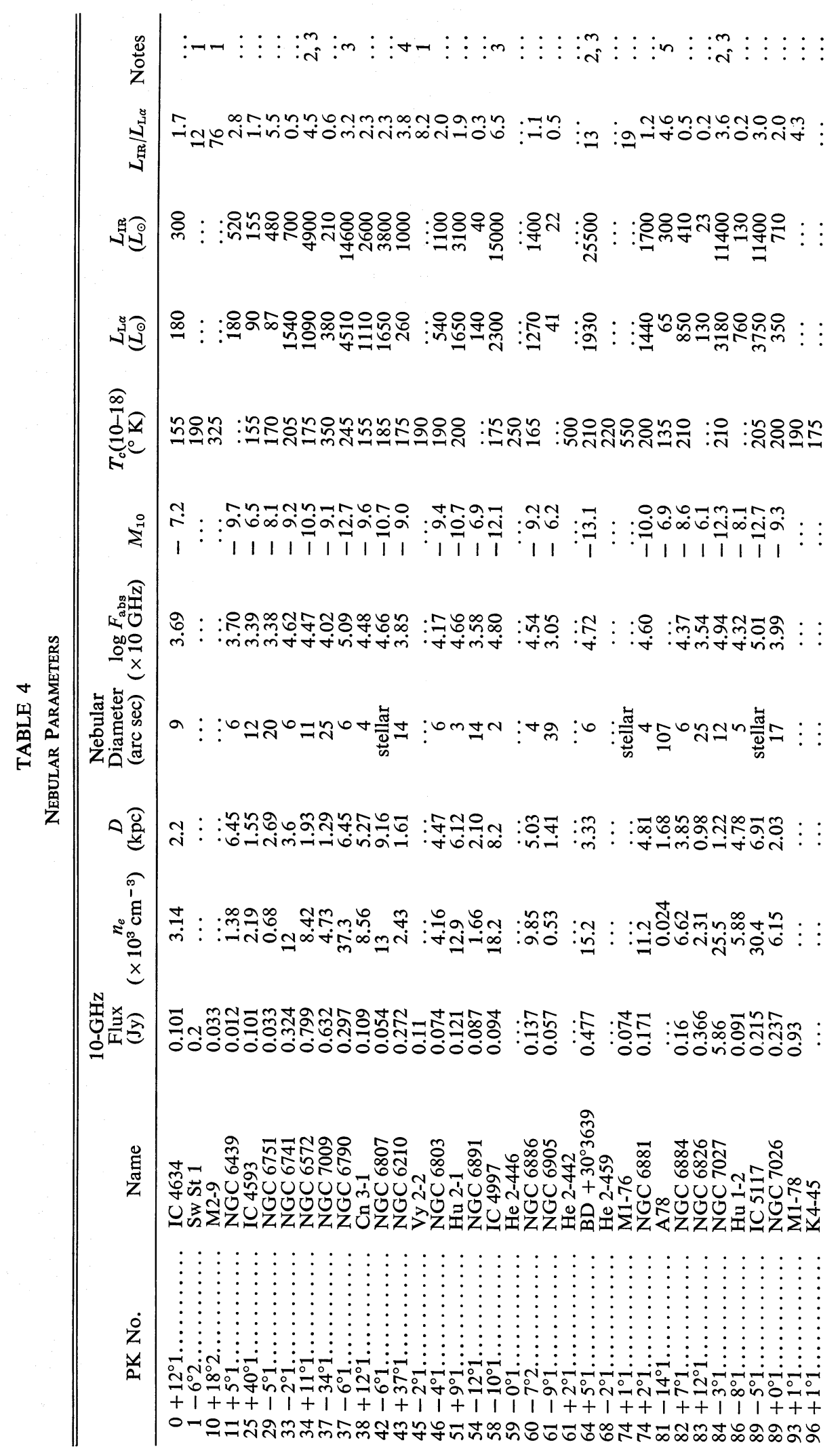

410 


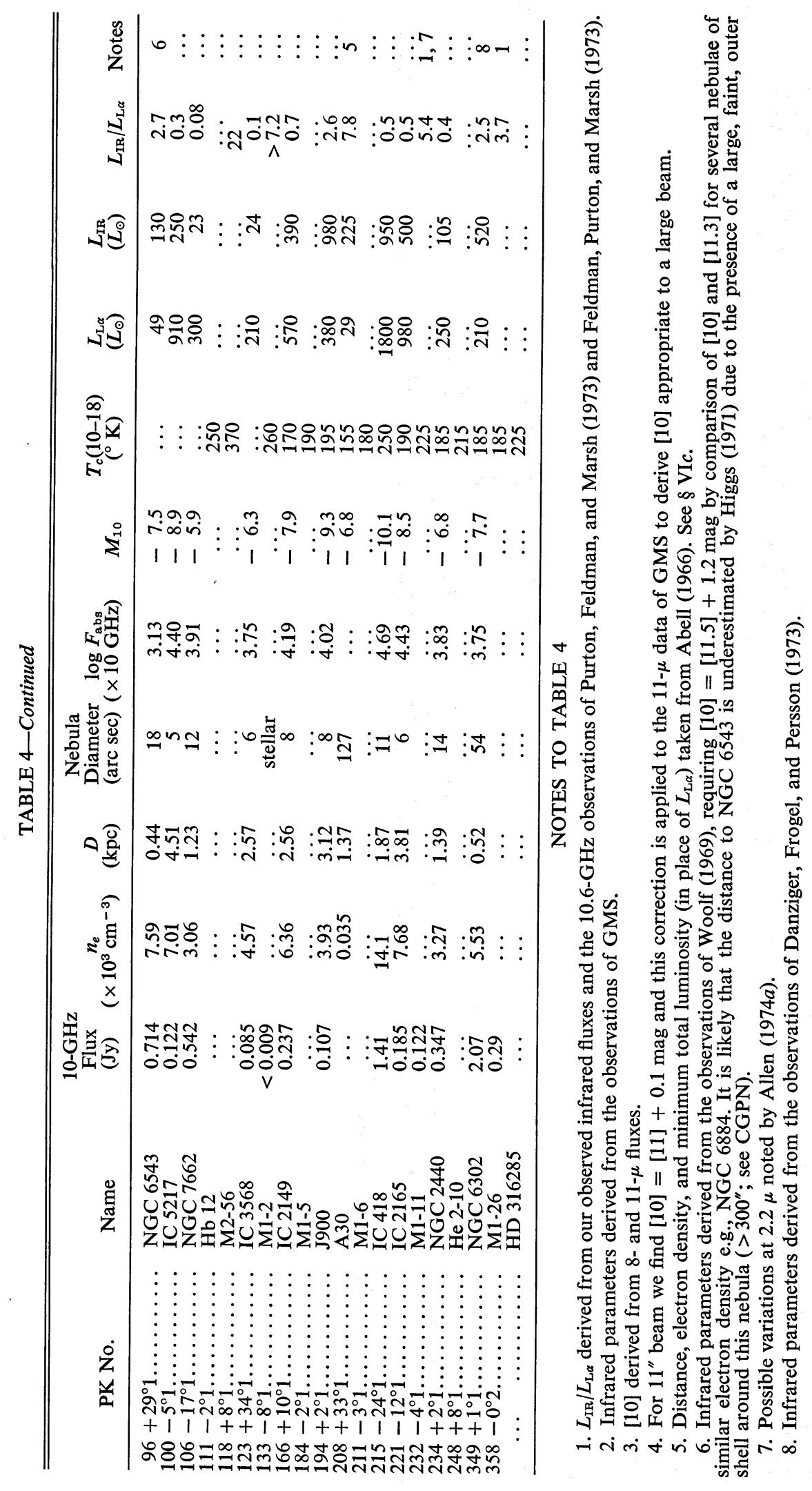

411 
If the only datum available was a $10-\mu$ magnitude, the integral beneath the curve of $F_{\lambda}$ was approximated by $\Delta \lambda$ multiplied by the value of $F_{\lambda}$ at $10 \mu$.

Also listed in table 4 is $T_{c}(10-18)$, the blackbody color temperature corresponding to the ratio of fluxes at 10 and $18 \mu$. Planetaries for which there are no distance estimates in the catalog of Higgs (1971) but for which values of $T_{c}(10-18)$ can be derived are included.

Table 4 lists the Perek and Kohoutek (1967) designation, the planetary name, the $10 \mathrm{GHz}$ flux in janskies, the electron density $n_{e}$ in $\mathrm{cm}^{-3}$, the distance $D$ in $\mathrm{kpc}$, the nebular diameter in arc seconds, $\log F_{\text {abs }}(10 \mathrm{GHz}), M_{10}, T_{c}(10-18), L_{\mathrm{L} \alpha}, L_{\mathrm{IR}}, L_{\mathrm{IR}} / L_{\mathrm{L} \alpha}$ (this ratio being independent of distance) and the footnote number if any.

\section{b) Heating Mechanisms}

As discussed in $\S$ III $c$, infrared emission appears to come from beyond the optical boundaries of some nebulae. This situation probably occurs in the early stages of planetary nebula evolution when not all of the ejected envelope has been fully ionized. For the majority of planetaries, however, it would appear that most of the infrared emission originates from within the ionized region of the nebula. There are no theoretical difficulties associated with the survival of dust grains in such ionized regions. Sputtering by thermal ions will be an insignificant destruction process, even for ice grains (Barlow 1971). Refractory grains such as are likely to be found in planetary nebulae (silicates, carbonates, graphite, etc.) are even more resistant to thermal sputtering.

$\mathrm{L} \alpha$ heating of dust grains was first suggested by Krishna Swamy and O'Dell (1968) as an energy source for the infrared emission observed from planetary nebulae. In the following discussion it will be assumed that all of the $\mathrm{L} \alpha$ photons resonantly trapped in the ionized region of a planetary nebula are absorbed by spherical dust grains uniformly mixed with the ionized gas.

In $1 \mathrm{~cm}^{3}$ the number of $\mathrm{L} \alpha$ photons created per second is $n_{e}{ }^{2} \alpha_{r}$ and in $1 \mathrm{~cm}^{3}$ the number of $\mathrm{L} \alpha$ photons destroyed per second is $n_{d} \sigma_{d} Q_{u} n_{\mathrm{L} \alpha} c$, where $n_{e}$ is the electron density, $\alpha_{r}$ is the proton recombination rate to levels with $n \geq 2, n_{d}$ is the grain number density, $\sigma_{d}$ is the grain cross-section, $Q_{u}$ is the ultraviolet emissivity of a grain, $n_{\mathrm{L} \alpha}$ is the L $\alpha$ photon number density, and $c$ is the velocity of light. If no $\mathrm{L} \alpha$ photons escape the nebula then

$$
n_{e}^{2} \alpha_{r}=n_{d} \sigma_{d} Q_{u} n_{\mathrm{L} \alpha} c
$$

If the grains are in equilibrium then the rate of emission of infrared radiation from the nebula will be equal to the total $\mathrm{L} \alpha$ grain heating rate

$$
F_{\mathrm{IR}}=n_{d} \sigma_{d} Q_{u} n_{\mathrm{L} \alpha}\left\langle\epsilon_{\mathrm{L} \alpha}\right\rangle c V
$$

where $F_{\mathrm{IR}}$ is the total nebular infrared luminosity,
$\left\langle\epsilon_{\mathrm{L} \alpha}\right\rangle$ is the energy of a $\mathrm{L} \alpha$ photon, and $V$ is the total volume of the nebula. Using equation (1) we have

$$
F_{\mathrm{IR}}=n_{e}{ }^{2} \alpha_{r}\left\langle\epsilon_{\mathrm{L} \alpha}\right\rangle V \text {. }
$$

With the assumption that all planetaries have the same mass of ionized gas,

$$
n_{e} V=\text { constant }
$$

and

$$
F_{\mathrm{IR}} \propto n_{e}
$$

The range of dust color temperatures encountered is sufficiently narrow so that for most nebulae the total infrared emission rate is directly proportional to $F_{\text {abs }}(10 \mu)$, the absolute flux at $10 \mu$,

i.e.,

$$
F_{\mathrm{IR}} \propto F_{\mathrm{abs}}(10 \mu)
$$

thus

$$
\log F_{\text {abs }}(10 \mu) \propto \log n_{e} ;
$$

and since $\quad M_{10} \propto-2.5 \log F_{\text {abs }}(10 \mu)$,

then

$$
M_{10}=-2.5 \log n_{e}+K_{1},
$$

where $K_{1}$ is a constant.

A correlation between $\log n_{e}$ and $M_{10}$ can be seen in figure 5. A line corresponding to the relation given

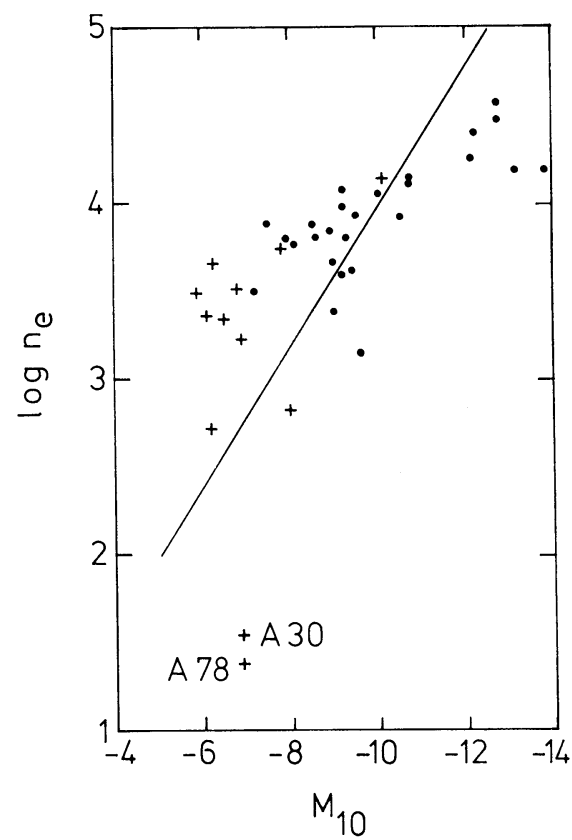

Fig. 5.-The plot of nebular electron density against absolute 10- $\mu$ magnitude for 38 nebulae. Nebulae smaller than our beam size are shown as dots and objects larger than our beams are shown as crosses. The line is drawn to illustrate the relation in eq. (4). 


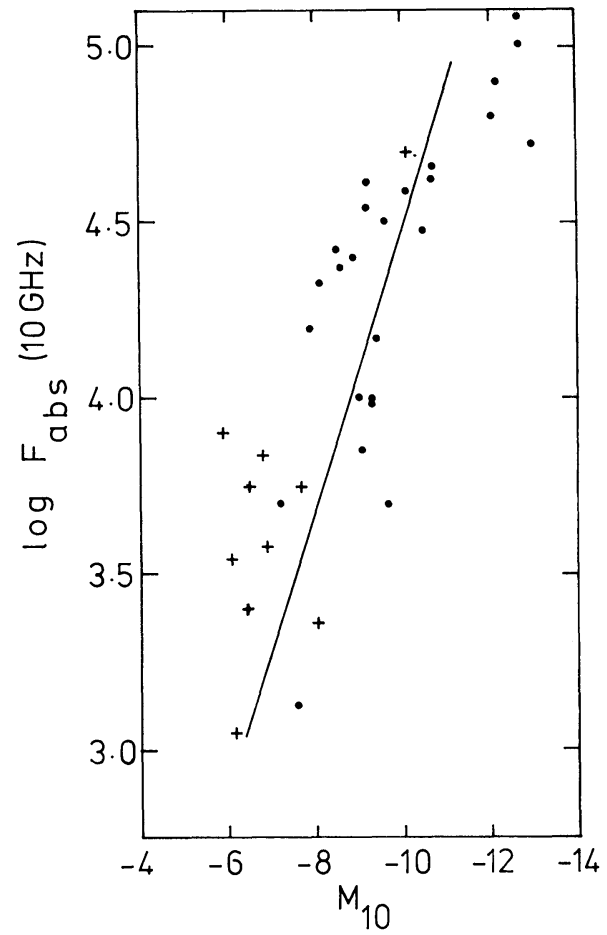

FIG. 6.-The plot of logarithmic absolute radio flux at $10 \mathrm{GHz}$ against absolute $10-\mu$ magnitude for 35 planetaries. Symbols are as in fig. 5. The line shows the relation in eq. (5).

by equation (4) has also been drawn on the figure. Ignoring the planetaries with $\log n_{e}<3$ (see $\S \mathrm{VI} c$ ) the deviation from this relation is probably due to the fact that the model of Higgs (1973) underestimates the densities of the densest and most compact planetaries due to their being optically thick in the Lyman continuum and consequently not fully ionized.

The absolute $10-\mathrm{GHz}$ flux from a planetary nebula due to free-free emission, $F_{\text {abs }}(10 \mathrm{GHz})$, is proportional to $n_{e}{ }^{2} V$ and, therefore, for constant ionized mass,

$$
F_{\text {abs }}(10 \mathrm{GHz}) \propto n_{e} .
$$

Using equation (4) we have

$$
M_{10}=-2.5 \log F_{\mathrm{abs}}\left(10 \mathrm{GHz}+K_{2}\right),
$$

where $K_{2}$ is a constant.

In figure 6 , the plot of $\log F_{\text {abs }}(10 \mathrm{GHz})$ versus $M_{10}$, a strong correlation can be seen. A line corresponding to the relation given by equation (5) has also been drawn showing good agreement with the observations.

The energy balance relation for one grain is given by

$$
4 Q_{i} \sigma T^{4}=Q_{u} n_{\mathrm{L} \alpha}\left\langle\epsilon_{\mathrm{L} \alpha}\right\rangle c
$$

where $Q_{i}$ is the infrared emissivity of the grain, $\sigma$ is

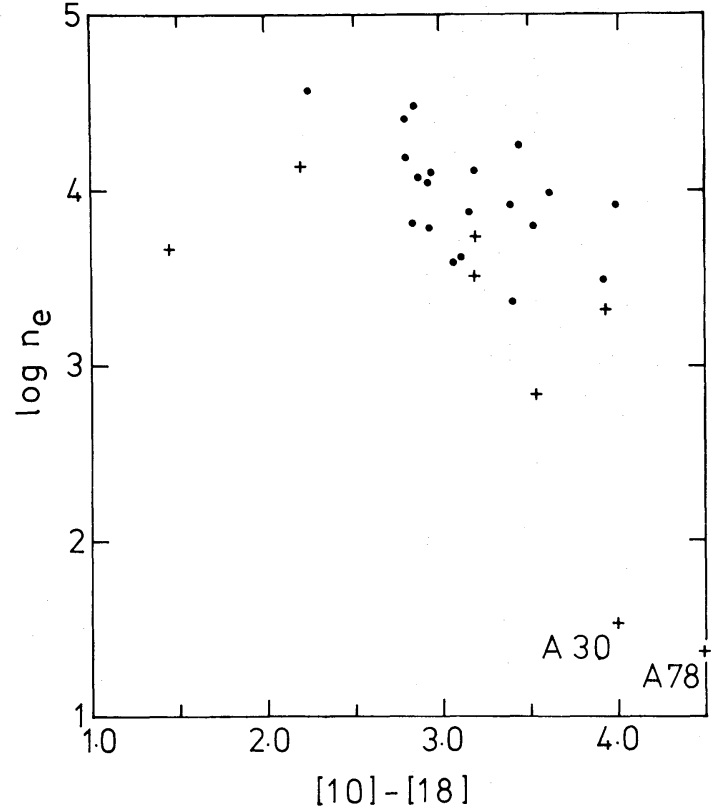

FIG. 7.-The trend of observed [10] - [18] color index of 28 planetaries with nebular electron density. Symbols as in fig. 5.

the Stefan-Boltzmann constant, and $T$ is the grain temperature. So using equation (1) we have

$$
T=n_{e}{ }^{1 / 2}\left(\frac{\left\langle\epsilon_{\mathrm{L} \alpha}\right\rangle \alpha_{r}}{4 n_{d} \sigma_{d} Q_{i} \sigma}\right)^{1 / 4}
$$

Figure 7 shows the trend of $T_{c}(10-18)$ with $\log n_{e}$ while figure 8 directly plots $n_{e}{ }^{1 / 2}$ versus $T_{c}(10-18)$ and

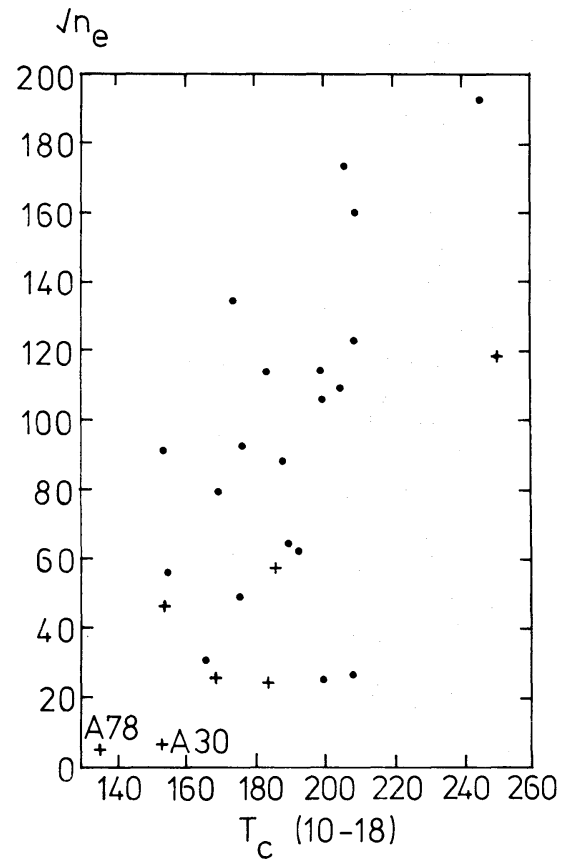

FIG. 8.-The plot of $\left(n_{e}\right)^{1 / 2}$ against color temperature between 10 and $18 \mu$ for 27 planetary nebulae; cf. eq. (7). 
shows a correlation with a large amount of scatter. There are probably several reasons for this scatter. The quantity $\left(n_{d} \sigma_{d}\right)$ may vary from nebula to nebula, as is likely also for the infrared emissivity $Q_{i}$. However, the dependence on these two quantities is weak. More significant perhaps could be the distortion of the derived color temperature $T_{c}(10-18)$ by the presence of features in the spectrum at 10 and/or $18 \mu$.

The infrared spectra of planetary nebulae which have been calculated by Terzian and Sanders (1972) are, in general, too cool when compared with the observations; this is probably due to their assumption that the dust grains reside outside the ionized gas.

The approximate mass of dust required to produce the observed infrared emission can be calculated from the relation

$$
M_{\mathrm{dust}}=\rho_{d}\left(\frac{4}{3} \pi a^{3}\right) \frac{L_{\mathrm{IR}}}{4 \pi a^{2} Q_{i} \sigma T^{4}},
$$

where $\rho_{d}$ is the density of the grain material and $\boldsymbol{a}$ is the radius of a grain. In the absence of knowledge of the infrared grain emissivity, $Q_{i}$ is taken to be equal to $2 \pi \boldsymbol{a} / \lambda$, where $\lambda$ is the wavelength corresponding to the peak of the infrared emission. This assumption leads to estimates of the mass in dust which are independent of grain radius. In this case $\lambda \simeq 20 \mu$. We take $\rho_{d}=3 \mathrm{~g} \mathrm{~cm}^{-3}$ and $T=T_{c}(10-18)$. For the planetary with the largest infrared luminosity, $\mathrm{BD}+30^{\circ} 3639$, we obtain

$$
M_{\text {dust }} \simeq 1.5 \times 10^{-4} M_{\odot} \text {, }
$$

which for an assumed nebular gas mass of $0.2 M_{0}$ leads to a dust-to-gas ratio of $\sim 1 / 1300$. A similar ratio is calculated for IC 4997, but for other planetaries the dust-to-gas ratios derived are less than this (usually a few times $10^{-5}$ ). These dust mass estimates might be increased by the use of more accurate values for the infrared emissivity $Q_{i}$. The dust-to-gas mass ratios derived here should be compared with average interstellar ratios of between $3 \times 10^{-3}$ and $10^{-2}$. The apparent smaller masses of dust in the older and less dense planetaries could be because any cooler dust, radiating at wavelengths much longer than $20 \mu$, has not been taken into account. However, there may well be real variations in the amount of dust present in planetaries, since several fairly dense nebulae have not been detected at $10 \mu$ (e.g., NGC 2392 and NGC 3242; see also $\S \mathrm{VI} c$ ).

Omitting planetaries with emission-line nuclei, inspection of the values of $L_{\mathrm{IR}} / L_{\mathrm{L} \alpha}$ listed in table 4 reveals that most nebulae can provide enough energy, in the form of $\mathrm{L} \alpha$ photons, to explain the observed infrared fluxes. Since the $\mathrm{L} \alpha$ photons absorbed by the grains are resonantly trapped in the nebula the amount of dust need not be very large, as demonstrated by Panagia and Ranieri (1973). Indeed for most planetaries large infrared excesses can be produced without any noticeable internal extinction in the visible region of the spectrum. This would be consistent with the observations by Wilson (1950) and Osterbrock (1973) of the intensities of the blue- and redshifted components of nebular emission lines.

A further consequence of heating of the dust by $\mathrm{L} \alpha$ photons would be that no radiation dilution effects should be seen; i.e., all grains, regardless of their position in the nebula, should have very nearly the same color temperature. This implies that the long-wavelength $(10-20 \mu)$ spectra of at least some nebulae should be characterized by a relatively narrow, blackbody-like, distribution rather than a flat $\lambda F_{\lambda}$ plot which is more suggestive of emission at a range of temperatures. Several nebulae for which $22-\mu$ points exist do seem to have a spectral turnover somewhere shortward of $18 \mu$ (except A30). Further, the 27- $\mu$ observations of three planetary nebulae by Jameson et al. (1974) indicate a continued decrease in $\lambda F_{\lambda}$ beyond $22 \mu$. These considerations further strengthen the case for $L \alpha$ heating as the dominant source of heating of grains.

However, consider a nebula in the earliest stages of evolution, when any grains left over from the preceding cool stellar phase would be mixed with a compact, partially ionized gas shell. These grains, together with any which might form by condensation during these evolutionary processes, would be absorbing appreciable quantities of nonionizing ultraviolet radiation and some Lyman continuum photons. A range of temperatures should therefore be present among the dust grains. It is believed that M1-2 may represent such a protoplanetary (O'Dell 1968). Figure 2 substantiates this picture of early evolution since M1-2 has a very flat spectrum.

\section{EMISSION-LINE NUCLEI AND DUST FORMATION}

\section{a) Emission-Line Nuclei}

A striking feature of our results is the correlation of detectable infrared fluxes with planetaries containing emission-line central stars from the list of Smith and Aller (1969). Of 30 nebulae with emission-line nuclei observed in the infrared, 19 have been detected at $10 \mu$. In contrast, five nebulae definitely containing absorption-line $\mathrm{O}$ stars have not been detected. The case for planetaries containing central stars with continuous spectra is more confused due to difficulties in accurately classifying such stars. However, some at least, such as NGC 6886, do show dust emission. Strong upper limits have been placed upon any possible dust emission from NGC 2392, a nebula containing a central star with a classical Of spectrum. A43, another nebula with an Of nucleus (Greenstein and Minkowski 1964), was also not detected. It is tempting to hypothesize that all planetaries with $L_{\mathrm{IR}} / L_{\mathrm{L} \alpha}$ significantly greater than 1 contain emission-line central stars. On this basis, one would expect that J900, NGC 6439, IC 5117, NGC 7027, NGC 6210, NGC 6790, NGC 6803, M1-26, M1-11, Vy 2-2, and M1-78 all contain emission-line stars. The converse of this conjecture, however, cannot hold since the Ofp nuclei seem to have $L_{\mathrm{IR}} / L_{\mathrm{L} \alpha} \leqslant 1$.

Table 5 displays data for some planetaries with emission-line nuclei classified by Smith and Aller (1969). The values of $L_{\mathrm{IR}} / L_{\mathrm{L} \alpha}$ show that the infrared 
TABLE 5

Ratio of InFrared Luminosity to L $\alpha$ LUMinosity For SOME Planetaries Having Emission-Line Nuclei

\begin{tabular}{|c|c|c|c|}
\hline Name & $\begin{array}{l}\text { Nucleus } \\
\text { Type }\end{array}$ & Diameter & $L_{\mathrm{IR}} / L_{\mathrm{L} \alpha}$ \\
\hline $\begin{array}{l}\text { NGC } 675, \ldots \ldots \ldots \ldots \\
\text { BD }+30^{\circ} 3639 \ldots \ldots \ldots \ldots\end{array}$ & $\begin{array}{l}\text { WC6 } \\
\text { WC9 }\end{array}$ & $\begin{array}{c}20^{\prime \prime} \\
6\end{array}$ & 13 \\
\hline 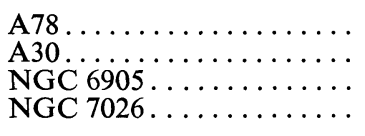 & $\begin{array}{l}\text { O vI } \\
\text { O VI } \\
\text { O VI } \\
\text { O VI }\end{array}$ & $\begin{array}{r}107 \\
127 \\
39 \\
17\end{array}$ & $\begin{array}{l}4.6 \\
7.8 \\
0.5^{*} \\
2.0\end{array}$ \\
\hline $\begin{array}{l}\text { IC } 418 \ldots \ldots \ldots \ldots \ldots \ldots \\
\text { IC } 2149 \ldots \ldots \ldots \ldots \ldots \ldots \\
\text { IC } 4593 \ldots \ldots \ldots \ldots \ldots\end{array}$ & $\begin{array}{l}\text { O7fp } \\
\text { O7.5fp } \\
\text { O7fp } \\
\text { O6fp }\end{array}$ & $\begin{array}{r}11 \\
8 \\
12 \\
25\end{array}$ & $\begin{array}{l}0.5 \\
0.7 \\
1.7 \\
0.2^{*}\end{array}$ \\
\hline $\begin{array}{l}\text { NGC } 6543 \ldots \ldots \ldots \ldots \ldots \\
\text { NGC } 6572 \ldots \ldots \ldots \ldots \ldots \\
\text { HD } 167362 \ldots \ldots \ldots \ldots \ldots \\
\text { IC } 4997 \ldots \ldots \ldots \ldots\end{array}$ & $\begin{array}{l}\text { W-Of } \\
\text { W-Of } \\
\text { W-Of } \\
\text { W-Of }\end{array}$ & $\begin{array}{r}18 \\
11 \\
1 \\
2\end{array}$ & $\begin{array}{l}2.7^{*} \\
4.5 \\
12 \\
6.5\end{array}$ \\
\hline $\begin{array}{l}\text { Cn 3-1 (VV 391) } \ldots \ldots \ldots \ldots \\
\text { Hu 2-1 (VV 458)........ }\end{array}$ & $\begin{array}{l}\mathrm{E} \\
\mathrm{E}\end{array}$ & $\begin{array}{l}4 \\
3\end{array}$ & $\begin{array}{l}2.3 \\
1.9\end{array}$ \\
\hline
\end{tabular}

* No 18- $\mu$ mag available.

emission from planetaries containing Ofp stars can be adequately powered by resonantly trapped $L \alpha$ radiation but that this mechanism would appear to be inadequate for certain nebulae with nuclei of the WolfRayet, O VI or WR-Of type. These particular objects are basically of two types: (1) compact, high-density nebulae; (2) several old, extended, very low-density nebulae. Each type will be discussed separately in the following sections.

\section{b) Compact High-Density Nebulae}

Examples of this type of object are BD $+30^{\circ} 3639$, HD 167362, and IC 4997. The fraction of radiation emitted shortward of the Lyman edge by early-type stars has been discussed by Panagia (1973). It is noted that all calculations of the flux emitted shortward of the Lyman edge are based on plane-parallel model atmospheres and that the effect of extended atmospheres (such as those encountered in emission-line stars) may be to increase the estimate of Lyman continuum flux by more than 50 percent, as found by Cassinelli (1971). This would imply that the values of $L_{\mathrm{L} \alpha}$ are underestimated for nebulae with emission-line nuclei, thus lessening the discrepancy with $L_{\mathrm{IR}}$. In addition, some compact planetaries may be so dense that they are self-absorbed even at $10 \mathrm{GHz}$, so that again their Lyman continuum luminosities will be underestimated.

Of the planetaries with nuclei of known spectral type, the largest value of $L_{\mathrm{IR}} / L_{\mathrm{L} \alpha}$ occurs for $\mathrm{BD}$ $+30^{\circ} 3639$, which contains one of the coolest known central stars, of spectral type WC 9. For an assumed effective temperature of just under $30,000^{\circ} \mathrm{K}$, less than 10 percent of the total stellar radiation is emitted shortward of the Lyman edge (Panagia 1973). Direct absorption by dust of stellar radiation from longward of the Lyman edge can therefore provide an adequate energy source for the infrared emission, with the corollary that the dust color temperature should decrease with increasing distance from the central star, in contrast to the case for $L \alpha$ heating where the color temperature should be reasonably uniform across the nebula.

The LTE unblanketed (plane-parallel) models of planetary nuclei by Hummer and Mihalas (1970) permit an evaluation of the role played by this nonionizing photon flux in heating grains. For a nucleus with $T_{\text {eff }}=40,000^{\circ} \mathrm{K}$ and $\log g=4.5$, the fractions of the total stellar continuum luminosity emitted shortward and longward of the Lyman edge, and in the form of $L \alpha$ photons (from Panagia 1973), are: $L(\lambda<912 \AA)=24$ percent; $L(\lambda>912 \AA)=76$ percent; $L_{\mathrm{L} \alpha}=18$ percent. For a somewhat hotter central star, of $T_{\text {eff }}=100,000^{\circ} \mathrm{K}$ and $\log g=5.0$, these become: $L(\lambda<912 \AA)=91$ percent; $L(\lambda>912 \AA)=$ 9 percent; $L_{\mathrm{L} \alpha}=29$ percent. Consequently, the ratio $L_{\mathrm{IR}} / L_{\mathrm{L} \alpha}$ can be as high as $\sim 5.0$ for a $40,000^{\circ} \mathrm{K}$ nucleus and up to $\sim 1.3$ for a $100,000^{\circ} \mathrm{K}$ star, if the grains absorb only photons longward of the Lyman edge with a constant absorption efficiency.

It is unlikely that many planetaries are dust limited (i.e., that dust, by absorbing a significant fraction of the stellar Lyman continuum, reduces the size of ionized zone). This is because the opacity of the neutral gas in the envelope to ionizing photons will be much greater than the opacity of the dust for the dust-to-gas ratios found in this study.

\section{c) A30, A78, and Dust Formation}

A30 and A78 are two extended planetary nebulae of low surface brightness (Abell 1966) containing central stars belonging to the $\mathrm{O}$ VI sequence (Smith and Aller 1969). Our detection of infrared radiation using a sky-chopping system implies a central concentration of emission around these nuclei. Section III $c$ deals with the emission profile across A30.

For the following reasons not too much faith should be placed in the values of $L_{\mathrm{IR}} / L_{\mathrm{L} \alpha}$ given for A30 and A78. (1) Only a fraction of the total infrared flux from these nebulae may have been detected with our $11^{\prime \prime}$ beams and the low color temperatures derived imply that considerable flux exists beyond the $20-\mu$ region. These factors should combine to increase significantly the infrared luminosity estimates. (2) Neither nebula has as yet been detected at radio wavelengths, so in place of $L_{\mathrm{L} \alpha}$ we have used Abell's (1966) estimates for the minimum luminosities of the nuclei. These luminosities were estimated using the distances and Zanstra minimum temperatures of the stars calculated on the assumption that the surface brightness of the nebulae on red plates is due predominantly to $\mathrm{H} \alpha$ emission. Greenstein and Minkowski (1964) note, however, that several plates of A78 taken with the 200 -inch $(5-\mathrm{m})$ telescope fail to show $\mathrm{H} \alpha$ or [N II] ( $\mathrm{H}$ is seen weakly in the spectra of the nuclei of A30 and A78 but all other elements seen in both the stars and nebulae are $\alpha$-particle nuclei). Derived distances, luminosities, and densities are therefore suspect. 
On figures 5 and 6 it can be seen that A30 and A78 depart significantly from the general trend. This might be ascribed to underestimates of the densities, although if $\mathrm{L} \alpha$ heating is to work and assuming that the grains are of the same nature as in other planetaries (which may not be the case), then densities $\geq 10^{3} \mathrm{~cm}^{-3}$ are required, which seems rather unlikely. Radio observations would be useful in determining some of these quantities. A 17th mag companion (separation 5"3) to the central star of A30 has been found by Cudworth (1973) and, if physically associated, might allow a distance estimate to be made.

The possibility that the dust seen in planetary nebulae is a remnant from some previous red giant or Mira phase can be ruled out for A30, A78, and many other planetaries since any such dust would have been swept to the rim of the nebula by the initial shell ejection. Another possibility which has been suggested (GMS; Hunter 1973) is that the dust condenses out during the expansion of the ejected envelope. While this may indeed be the case for some planetaries, the dust seen at the centers of A30 and A78 cannot have had this origin. Apart from the fact that dust formation in an expanding envelope ought not to produce an apparent central concentration of emission, the effect of the high-velocity mass outflow from the central star on any dust which may have been left at the center of the nebula must be considered. Although sputtering by thermal ions will have an insignificant effect on dust grains in planetary nebulae due to the extremely low sputtering efficiencies at thermal energies (Barlow 1971), this will not be the case for sputtering by ions in the high-velocity stellar wind from an emission-line star. The width of the emission lines in the $\mathrm{O}$ VI nuclei of A30 and A78 indicate velocities of the order of 1000 $\mathrm{km} \mathrm{s}^{-1}$ (Greenstein and Minkowski 1964; Smith and Aller 1969). This velocity corresponds to an energy of $20 \mathrm{keV}$ for a helium ion in the outflow. The sputtering yield of materials has a broad maximum in this energy range and lifetimes of graphite and silicate grains in the central regions of A30 and A78 have been calculated using the appropriate experimentally determined sputtering yields (the number of lattice particles ejected per incident ion) of 0.05 for helium incident on graphite and 0.1 for helium on stony silicate materials (see Rosenberg and Wehner 1962; Wehner, KenKnight, and Rosenberg 1963). Inclusion of the effects of sputtering by heavier ions (and by hydrogen if any) will approximately double the sputtering rate due to helium alone. At a distance of $5 \times 10^{16} \mathrm{~cm}$ from a central star which is losing mass at a rate of $10^{-6} M_{\odot}$ year ${ }^{-1}$, graphite particles of radius $2 \times 10^{-6} \mathrm{~cm}$ will be destroyed in 1000 years and silicate particles of radius $5 \times 10^{-6} \mathrm{~cm}$ will be destroyed in 150 years (the life time of carbonate particles will be even shorter).

These times are of the same order, or less than, the time required to sweep stationary grains to the same velocity as the wind (by the kinetic pressure of the wind) and are much shorter than the likely duration of the emission-line phase of the central star. If the grains are not to be destroyed or swept away they must be continuously replenished and be moving at the same velocity as the wind. We therefore conclude that the dust grains are forming in the high-velocity mass outflow from the central star.

Dust formation around hot stars has also been suggested by Cohen and Barlow (1973) for certain Population I Of stars and by Allen and Penston (1973) for Be stars. The latter note a negative correlation between stellar and dust temperatures, and it is interesting that the coolest color temperature found in our survey $\left(136^{\circ} \mathrm{K}\right)$ is in $\mathrm{A} 78$, which contains one of the hottest known nuclei, with an effective temperature in excess of $100,000^{\circ} \mathrm{K}$ (Smith and Aller 1969). The theoretical problems associated with the condensation of dust around such hot stars are, needless to say, quite significant. Assuming that high-temperature ions cannot directly condense into grains, some sort of adiabatic decoupling of the gas would seem to be required, presumably by means of instabilities in the flow.

High-sensitivity infrared observations of A30 and A78 are needed at wavelengths shorter than $8.6 \mu$ in order to determine the temperature of the hottest and presumably most recently formed dust.

Dust formation in the mass-loss outflows from emission-line nuclei of planetary nebulae would explain the correlation observed between the existence of an infrared excess and the presence of such emissionline central stars and would explain also the lack of significant 10 - or $18-\mu$ radiation from nebulae with absorption-line $O$ stars, since these stars are not thought to be undergoing mass loss. The question of whether dust also forms during the initial envelope ejection in planetary nebula remains to be resolved. One might speculate, however, that dust formation only during emission-line nucleus phases (at the normal interstellar dust-to-gas ratio) would provide a natural explanation for the low apparent ratios inferred, since the dust concentration would be considerably diluted by the gas originally ejected in the planetary envelope.

Osterbrock (1968) has estimated that planetary nebulae may return to the interstellar medium about one-third of the total amount of gas contributed to the medium each year. The evidence for dust grains within the nebulae suggests that the planetaries will add to the total dust mass of the interstellar medium. However, if the low dust-to-gas mass ratios for planetary nebulae derived here are correct then it is unlikely that they act as a significant source of interstellar dust grains. This is because mantle growth will not take place in average interstellar cloud conditions (Barlow 1974), with the result that dust grains from planetaries cannot increase their mass concentration toward average interstellar values. Planetary nebulae should therefore act to dilute the interstellar dust concentration rather than enrich it.

\section{CONCLUSIONS}

1. An appreciable body of homogeneous observations of planetary nebulae is presented, principally at 10 and $18 \mu$, which enables comparisons to be made 
between various physical parameters of these objects.

2. Many nebulae are detected at these long wavelengths which are either very faint or undetected at near-infrared wavelengths. Large fluxes of radiation are common at $10 \mu$, and even larger ones are found from a number of nebulae at $18 \mu$, so that steeply rising spectra are common.

3. Some apparent emission features near $10 \mu$ are seen in nebulae for which narrow-band data are available.

4. A variety of arguments are adduced to show that this long-wavelength radiation is either predominantly or almost entirely continuum emission.

5. The fluxes at 10 and $18 \mu$ are substantially greater than those to be expected from an extrapolation of free-free emission from the radio region, although this mechanism may account for the radiation at shorter wavelengths.

6. The steeply rising spectra coupled with apparent features near $10 \mu$ are strongly suggestive of thermal emission by dust grains.

7. A model is proposed whereby dust grains coexist with the ionized gases within the nebulae, and are heated by the absorption of $\mathrm{L} \alpha$ photons resonantly trapped in the ionized region. An observational consequence of this type of heating for the energy distributions is discussed and evidence which vindicates the mechanism is found.

8. The model predicts several relationships between physical parameters of the nebulae which are in substantial agreement with the findings. These relationships are: (i) nebular absolute magnitudes at $10 \mu \propto$ $\log n_{e}$; (ii) nebular absolute magnitudes at $10 \mu \propto \log$ (intrinsic radio flux at $10 \mathrm{GHz}$ ); and (iii) nebular color temperatures between 10 and $18 \mu \propto\left(n_{e}\right)^{1 / 2}$.

9. Nebular $L \alpha$ luminosities are computed from the radio fluxes and compared with the infrared luminosities. In some nebulae, nonionizing continuum ultraviolet radiation longward of the Lyman edge may be needed to power the dust emission in addition to $\mathrm{L} \alpha$ photons.

10. Low values of the dust-to-gas ratio are found in the nebulae.

11. Of particular importance, two very large, lowsurface-brightness nebulae (A30 and A78) containing very hot nuclei are detected at 10 and $18 \mu$. The radiation in A30 is very strongly concentrated near the nucleus-no detectable emission is found elsewhere along two nebular diameters through the central star.

12. Arguing principally from these two nebulae, it is suggested that the dust grains must actually form in the high-velocity mass outflows from the emission-line nuclei.

It is a pleasure to thank Drs. N. J. Woolf and W. A. Stein for observing time at Mount Lemmon, Drs. D. A. Allen, H. M. Johnson, and A. E. Wright for kindly sending us data in advance of publication, and Drs. D. P. Gilra and F. C. Gillett for useful discussions. Infrared astronomy at Berkeley is supported by the National Science Foundation under grant GP-31592X. M. J. B. is grateful for the award of an ESRO-NASA fellowship while at Berkeley.
Abell, G. O. 1966, Ap. J., 144, 259.

Allen, D. A. 1973, M.N.R.A.S., 161, 145. $1974 a$, preprint. $1974 b$, private communication

Allen, D. A., and Glass, I. S. 1974, preprint.

Allen, D. A., and Penston, M. V. 1973, M.N.R.A.S., 165, 121. Allen, D. A., and Swings, J. P. 1972a, Ap. J., 174, 583. $1972 b$, Ap. Letters, $10,83$.

Aller, L. H. 1968, in IAU Symposium No. 34, Planetary Nebulae, ed. D. E. Osterbrock and C. R. O'Dell (Dordrecht: Reidel), p. 339.

Aller, L. H., and Walker, M. F. 1970, Ap. J., 161, 917.

Andrillat, Y. 1968, in IAU Symposium No. 34, Planetary Nebulae, ed. D. E. Osterbrock and C. R. O'Dell (Dordrecht: Reidel), p. 63.

Arhipova, V. P. 1973, 18th Liège Colloquium, Les Nebuleuses Planétaires (Mém. Soc. Roy. Sci. Liège), p. 477.

Barlow, M. J. 1971, Nature Phys. Sci., 232, 152.

- .1974 , in preparation.

Bidelman, W. P., and Krumenaker, L. E. 1972, Pub. A.S.P., 84, 685 .

Blanco, V., Kunkel, W., and Hiltner, W. A. 1968, Ap. J. (Letters), 152, L137.

Blanco, V., Kunkel, W., Hiltner, W. A., Lynga, G., Bradt, H., Clark, G., Naranan, S., Rappaport, S., and Spada, G. 1968, Ap. J., 152, 1015.

Cassinelli, J. 1971, Ap. Letters, 8, 105.

Cohen, M. 1973a, M.N.R.A.S., 161, 85. 1973b, ibid., 164, 395.

Cohen, M., and Barlow, M. J. 1973, Ap. J. (Letters), 185, L37. 1974, in preparation.

Cudworth, K. M. 1973, Pub. A.S.P., 85, 401.

Danziger, I. J., Frogel, J. A., and Persson, S. E. 1973, Ap. J. (Letters), 184, L29.
REFERENCES

Feldman, P. A., Purton, C. R., and Marsh, K. A. 1973, Nature Phys. Sci., 245, 7.

Gillett, F. C., Forrest, W. J., and Merrill, K. M. 1973, Ap. J., $183,87$.

Gillett, F. C., Knacke, R. F., and Stein, W. A. 1971, Ap. J. (Letters), 163, L57.

Gillett, F. C., Merrill, K. M., and Stein, W. A. 1972, Ap. J., 172, 367 (GMS)

Gillett, F. C., and Stein, W. A. 1969, Ap. J. (Letters), 155, L97.

Greenstein, J. L. 1972, $A p . J .$, 173, 367.

Greenstein, J. L., and Minkowski, R. 1964, Ap. J., 140, 1601.

Herbig, G. H., and Boyarchuk, A. A. 1968, Ap. J., 153, 397.

Herbig, G. H., and Flannery, B. P. 1973, Ap. J., 183, 491.

Higgs, L. A. 1971, Catalog of Radio Observations of Planetary Nebulae and Related Optical Data (Pub. Ap. Branch, N.R.C., Canada), Vol. 1, No. 1.

- . 1973, M.N.R.A.S., 161, 313.

Hummer, D. G., and Mihalas, D. 1970, M.N.R.A.S., 147, 339.

Hunter, J. H. 1973, Ap. J., 180, 99.

Jameson, R. F., Longmore, A. J., McLinn, J. A., and Woolf, N. J. 1974, submitted to $A p$. J.

Johnson, H. M. 1973, 18th Liège Colloquium, Les Nebuleuses Planétaires, p. 121.

Khromov, G. S., and Moroz,V.I. 1972, Soviet Astr.-AJ, 15, 892.

Kohoutek, L. 1968, in IAU Symposium No. 34, Planetary Nebulae, ed. D. E. Osterbrock and C. R. O'Dell (Dordrecht: Reidel), p. 324.

- 1972, Astr. and Ap., 16, 291.

Kostjakova, E. V., Arhipova, V. P., and Savel'eva, M. V. 1973, 18th Liège Colloquium, Les Nebuleuses Planétaires, p. 473.

Kostjakova, E. V., Savel'eva, M. V., Dokuchaeva, O. D., and Noskova, R. I. 1968, in IAU Symposium No. 34, Planetary Nebulae, ed. D. E. Osterbrock and C. R. O'Dell (Dordrecht: Reidel), p. 317. 
Krishna Swamy, K. S., and O'Dell, C. R. 1968, Ap.J. (Letters), 151, L61.

Lee, P., Aller, L. H., Czyzak, S. J., Duvall, R. H. 1969, Ap.J., 155, 853.

Lee, T. A. 1968, Ap. J., $152,913$.

Merrill, K. M. 1973, private communication.

Minkowski, R. 1974, private communication.

O'Dell, C. R. 1968, in IAU Symposium No. 34, Planetary Nebulae, ed. D. E. Osterbrock and C. R. O'Dell (Dordrecht: Reidel), p. 361.

Osterbrock, D. E. 1968, Ann. Rev. Astr. and Ap., $2,95$.

- 1973, Bull. A.A.S., 5, 423.

Panagia, N. 1973, A.J., 78, 929.

Panagia, N., and Ranieri, M. 1973, 18th Liège Colloquium, Les Nebuleuses Planétaires, p. 275.

Perek, L., and Kohoutek, L. 1967, Catalog of Planetary Nebulae (Prague: Czechoslovak Institute of Sciences) (CGPN).

Persson, S. E., and Frogel, J. A. 1973, Ap. J., 182, 503 (PF).

Purton, C. R., and Feldman, P. A. 1974, preprint.
Purton, C. R., Feldman, P. A., and Marsh, K. A. 1973, Nature Phys. Sci., 245, 6.

Rosenberg, D., and Wehner, G. K. 1962, J. Appl. Phys., 33, 1842.

Rubin, R. H. 1968, Ap. J., 154, 391.

- 1970, Astr. and Ap. 8, 171.

Sanduleak, N. 1972, Ap. J.(Letters), 164, L71.

Sanduleak, N., and Stephenson, C. B. 1972a, Ap. J., 178, 183. - 1972b, Pub. A.S.P., 84, 816.

Shao, C.-Y., and Liller, W. 1974, preprint.

Smith, L. F., and Aller, L. H. 1969, Ap. J., 157, 1245.

Terzian, Y., and Sanders, D. 1972, A.J., 77, 350.

Wehner, G. K., KenKnight, C., and Rosenberg, D. L. 1963, Planet. and Space Sci., 11, 885.

Willner, S., Becklin, E., and Visvanathan, N. 1972, Ap.J., 175, 699 (WBV).

Wilson, O. C. 1950, Ap. J., 111, 729.

Woolf, N. J. 1969, Ap. J. (Letters), 157, L37.

——. 1973, Ap. J., 185, 229.

Michael J. Barlow: Astronomy Centre, University of Sussex, Falmer, Brighton BN1 9QH, England

MARTIn CoHen: Berkeley Astronomy Department, University of California, Berkeley, CA 94720 